\title{
A STUDY OF ASSESSMENT IN PERIPHERAL NEUROPATHY IN PATIENTS WITH NEWLY DETECTED THYROID DISORDERS IN A TERTIARY CARE TEACHING INSTITUTE
} \author{
Jeeja Janardhanan6, Saravanamoorthy Magalingam7, Sindhiya Jayachandran ${ }^{8}$ \\ ${ }_{1}^{1}$ Professor, Department of Medicine, Government Stanley Medical College, Chennai. \\ ${ }^{2}$ Assistant Professor, Department of Medicine, Government Stanley Medical College, Chennai. \\ ${ }^{3}$ Post Graduate, Department of Medicine, Government Stanley Medical College, Chennai. \\ ${ }^{4}$ Assistant Professor, Department of Medicine, Government Stanley Medical College, Chennai. \\ ${ }^{5}$ Post Graduate, Department of Medicine, Government Stanley Medical College, Chennai. \\ ${ }^{6}$ Post Graduate, Department of Medicine, Government Stanley Medical College, Chennai. \\ 7 Post Graduate, Department of Medicine, Government Stanley Medical College, Chennai. \\ sPost Graduate, Department of Medicine, Government Stanley Medical College, Chennai.
}

Rajan Ganesan'1, Marimuthu Arumugam², Arungandhi Pachaiappan³, Thilakavathi Rajendran4, Archana Balakrishnan Mani5,

ABSTRACT
BACKGROUND
Thyroid dysfunction is associated with characteristic symptoms, signs and functional alterations in many organs and systems.
The purpose of this study was to evaluate objectively the functional changes in the peripheral nervous system and to determine the
type and the prevalence of these diseases. The prevalence of neuromuscular disorders related to thyroid dysfunction has
been reported to be between $20-80 \%$.

\section{AIMS AND OBJECTIVES}

- To study the correlation and prevalence of nerve conduction abnormalities in patients with thyroid dysfunction.

- $\quad$ To determine the type of peripheral neuropathy in these patients.

\section{MATERIALS AND METHODS}

This study was carried out in Medicine OPD and Endocrinology OPD over a period of 8 months; 50 consecutive patients with newly detected thyroid dysfunction and who fulfilled the inclusion criteria were included in the study and the required data were recorded from each subject with an in-person interview by administering a specific questionnaire. Nerve Conduction Study was performed in all these subjects by using the Standard RMS ENMG EP MARK II machine and results were correlated.

\section{RESULTS}

Out of the 50 patients studied 18 patients had neuropathy, of which 14 of them were hypothyroid and 4 of them had hyperthyroidism; 2 patients had mononeuropathy and 16 of them had polyneuropathy. Carpal tunnel syndrome was present in 2 individuals who had hypothyroidism; 14 patients had predominantly sensory neuropathy and 4 individuals had both sensory and motor polyneuropathy. The most common neurological abnormalities detected were sensory axonal polyneuropathy, mononeuropathy involving the sural nerve, mononeuritis multiplex pattern, carpal tunnel syndrome.

\section{CONCLUSION}

This study concludes that hypothyroid patients are more prone to develop neuropathy predominantly involving the sensory nerves of both lower limbs. This study clearly depicts that peripheral nervous system involvement is more often seen in patients with newly detected thyroid dysfunction. Electrophysiological studies help us in the diagnosis of this type of subclinical polyneuropathy. So nerve conduction studies can be included in the early part of diagnostic workup panel in newly detected thyroid illness.

\section{KEYWORDS}

Hypothyroidism, Hyperthyroidism, Nerve Conduction Abnormalities, Neuropathy.

HOW TO CITE THIS ARTICLE: Ganesan R, Arumugam M, Pachaiappan A, et al. A study of assessment in peripheral neuropathy in patients with newly detected thyroid disorders in a tertiary care teaching institute. J. Evolution Med. Dent. Sci. 2016;5(54):36713682, DOI: $10.14260 /$ jemds/2016/845

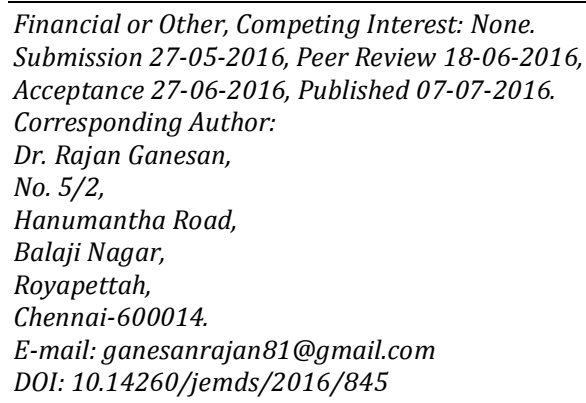

\section{INTRODUCTION}

Thyroid dysfunction is associated with characteristic symptoms, signs and functional alterations in many organs and systems. Peripheral nervous system is one amongst the various organ systems affected by thyroid dysfunction. Two types of peripheral neuropathy are reported in hypothyroid patients. They are Sensorimotor polyneuropathy and Mononeuropathy. Neuropathy is found to be uncommon in hyperthyroidism. The prevalence of neuromuscular disorders related to thyroid dysfunction has been reported to be between $20-80 \%$. Of this, $40 \%$ of patients with 
hypothyroidism have sensorimotor neuropathy early in the course of illness. Carpal tunnel syndrome is also commonly seen. Hence, this study was carried out to evaluate objectively the functional changes in the peripheral nervous system and to determine the type and the prevalence of these diseases in patients with newly detected thyroid dysfunction.

\section{AIMS AND OBJECTIVES}

- To study the correlation and prevalence of nerve conduction abnormalities in patients with newly detected thyroid dysfunction.

- To determine the type of peripheral neuropathy in these patients.

\section{MATERIALS AND METHODS}

After due Institutional Ethical Committee approval, this study was carried out in Medicine OPDs and Endocrinology OPD over a period of 8 months; 50 consecutive patients with newly detected thyroid dysfunction including both hypothyroidism and hyperthyroidism were included in the study. The following criteria were used to detect the same.

\begin{tabular}{|c|c|c|c|}
\hline Thyroid tuncionol stote & ТSH $10.3-3.3 \mathrm{~m} / \mathrm{M}$ & Free I, $10-30 \mathrm{~mol} / \mathrm{d}$ & 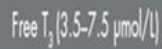 \\
\hline EUTHYROID & Nomol & Namd & Nomol \\
\hline HYPERTHYROID & Undetectoble & High & High \\
\hline HYPOTHYROID & High & low & low \\
\hline
\end{tabular}

Fig. 1: Interpretation of Thyroid Function Tests

Patients who were on Anti-cancer medications, AntiRetrovirals, Metronidazole and other drugs causing neuropathy were excluded from the study. Patients with Diabetes Mellitus, Chronic Alcoholism and other comorbid illnesses that would predispose to neuropathy like nutritional deficiency, connective tissue disorders, liver failure and kidney failure were not included in the study. Patients who were in Intensive Care Units and those who were pregnant were excluded from the study.

The required data were recorded from each subject with an in-person interview by administering a specific questionnaire. Nerve Conduction Study was performed in all these subjects by using the Standard RMS ENMG EP MARK II machine. The Latency, Amplitude, duration and velocity of motor and sensory nerves were studied. Motor Nerve Conduction Velocity (MNCV) was evaluated by Belly Tendon montage. Sensory Nerve Conduction Velocity (SNCV) was measured by anti-dromic stimulation. The results obtained were interpreted and statistical analysis was done.

\section{STATISTICAL ANALYSIS AND RESULTS}

Descriptive statistics was done for all data and were reported in terms of mean values and percentages. Suitable statistical tests of comparison were done. Continuous variables were analysed with the unpaired ' $t$ ' test. Categorical variables were analysed with the Chi-Square test and Fisher Exact Test. Statistical significance was taken as $\mathrm{P}<0.05$. The data was analysed using SPSS version 16 and Microsoft Excel 2007.

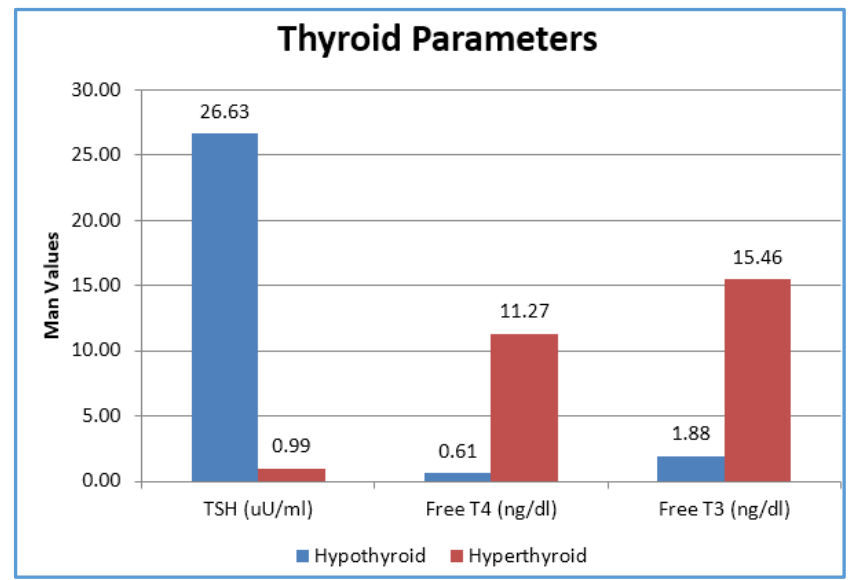

Fig. 2: Mean Values of TSH, Free T4, Free T3 among the Study Population

\begin{tabular}{|c|c|c|c|c|}
\hline \multicolumn{2}{|c|}{$\begin{array}{c}\text { Thyroid } \\
\text { Parameters }\end{array}$} & $\begin{array}{c}\text { TSH } \\
(\mathrm{uU} / \mathrm{mL})\end{array}$ & $\begin{array}{l}\text { Free T4 } \\
\text { (ng/dL) }\end{array}$ & $\begin{array}{l}\text { Free T3 } \\
\text { (ng/dL) }\end{array}$ \\
\hline \multirow{3}{*}{ All } & $\mathrm{N}$ & 50 & 50 & 50 \\
\hline & $\begin{array}{l}\mathrm{Me} \\
\text { an }\end{array}$ & 19.96 & 3.39 & 5.41 \\
\hline & SD & 26.05 & 5.58 & 6.98 \\
\hline \multirow{3}{*}{$\begin{array}{l}\text { Hypothyr } \\
\text { oid }\end{array}$} & $\mathrm{N}$ & 37 & 37 & 37 \\
\hline & $\begin{array}{l}\mathrm{Me} \\
\text { an }\end{array}$ & 26.63 & 0.61 & 1.88 \\
\hline & SD & 27.31 & 0.22 & 0.50 \\
\hline \multirow{3}{*}{$\begin{array}{l}\text { Hyperthy } \\
\text { roid }\end{array}$} & $\mathrm{N}$ & 13 & 13 & 13 \\
\hline & $\begin{array}{l}\mathrm{Me} \\
\text { an }\end{array}$ & 0.99 & 11.27 & 15.46 \\
\hline & SD & 2.69 & 5.99 & 7.12 \\
\hline & & $\begin{array}{l}\text { Iues of } T \\
\text { Study }\end{array}$ & $\begin{array}{l}\text { Te T4, Frt } \\
\text { ation }\end{array}$ & mong the \\
\hline
\end{tabular}

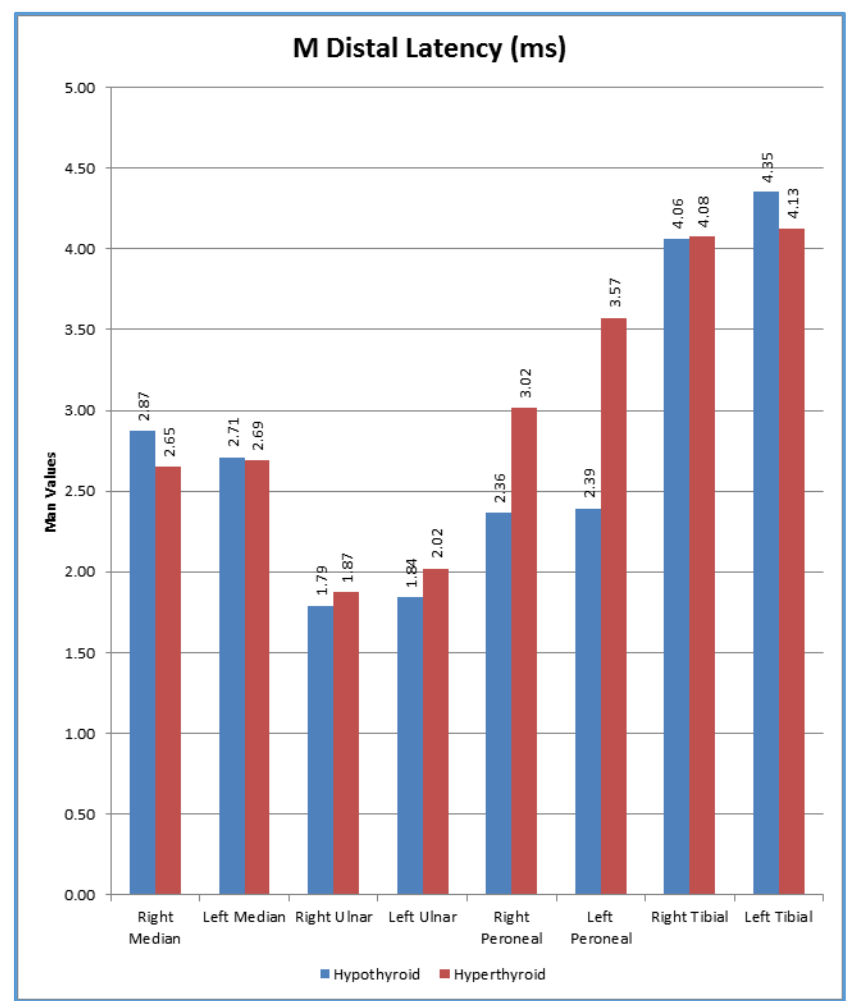

Fig. 3: Bar Diagram showing Motor Distal Latencies in Nerves of Upper and Lower Limbs 


\begin{tabular}{|c|c|c|c|c|c|c|c|c|c|}
\hline \multicolumn{2}{|c|}{$\begin{array}{c}\text { M Distal } \\
\text { Latency (ms) }\end{array}$} & \multirow{2}{*}{ 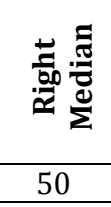 } & \multirow{2}{*}{ 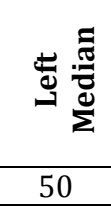 } & \multirow{2}{*}{ 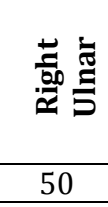 } & \multirow{2}{*}{ 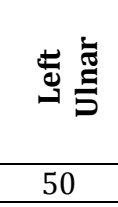 } & \multirow{2}{*}{ 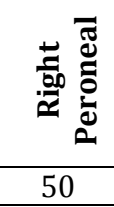 } & \multirow{2}{*}{ 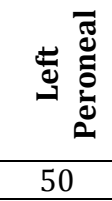 } & \multirow{2}{*}{ 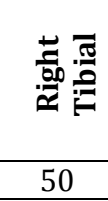 } & \multirow{2}{*}{$\begin{array}{l}\text { 离 } \\
50 \\
50\end{array}$} \\
\hline \multirow{3}{*}{ All } & $\mathrm{N}$ & & & & & & & & \\
\hline & Mean & 2.81 & 2.70 & 1.81 & 1.89 & 2.53 & 2.70 & 4.07 & 4.29 \\
\hline & SD & 0.60 & 0.64 & 0.59 & 0.56 & 0.85 & 0.98 & 0.51 & 0.59 \\
\hline \multirow{3}{*}{ Hypothyroid } & $\mathrm{N}$ & 37 & 37 & 37 & 37 & 37 & 37 & 37 & 37 \\
\hline & Mean & 2.87 & 2.71 & 1.79 & 1.84 & 2.36 & 2.39 & 4.06 & 4.35 \\
\hline & SD & 0.68 & 0.71 & 0.61 & 0.58 & 0.74 & 0.84 & 0.50 & 0.60 \\
\hline \multirow{3}{*}{ Hyperthyroid } & $\mathrm{N}$ & 13 & 13 & 13 & 13 & 13 & 13 & 13 & 13 \\
\hline & Mean & 2.65 & 2.69 & 1.87 & 2.02 & 3.02 & 3.57 & 4.08 & 4.13 \\
\hline & SD & 0.26 & 0.41 & 0.55 & 0.47 & 0.99 & 0.80 & 0.55 & 0.56 \\
\hline \multicolumn{2}{|c|}{ P value Unpaired ' $\mathrm{t}$ ' Test } & 0.1001 & 0.9118 & 0.6554 & 0.2836 & 0.2449 & 0.1123 & 0.9412 & 0.2347 \\
\hline
\end{tabular}

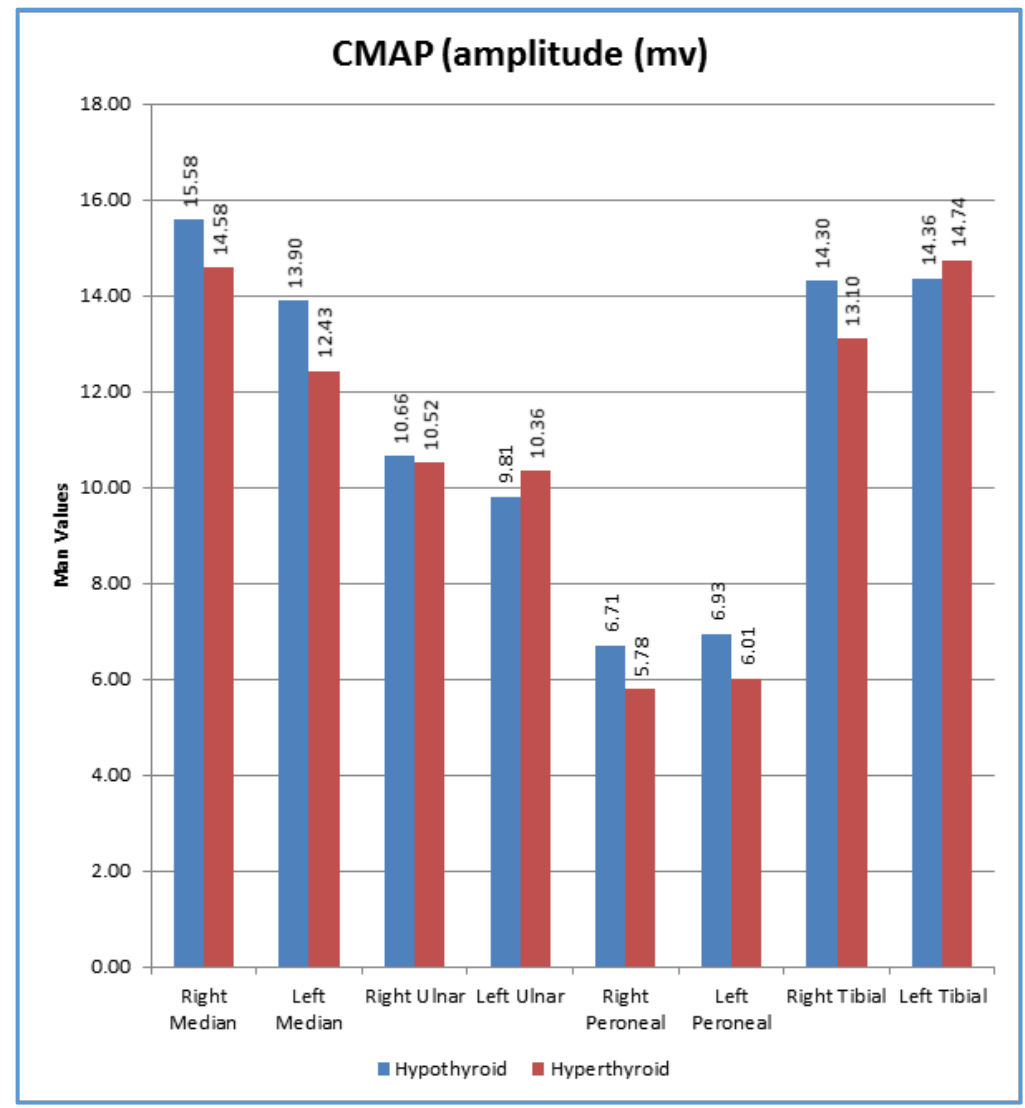

Fig. 4: Compound Muscle Action Potential (CMAP) Bar Diagram Representing Mean CMAP Values of Both Upper and Lower Limb Nerves

\begin{tabular}{|c|c|c|c|c|c|c|c|c|c|}
\hline \multicolumn{2}{|c|}{$\begin{array}{c}\text { CMAP } \\
\text { (Amplitude (mv)) }\end{array}$} & 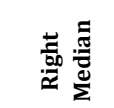 & 类 & 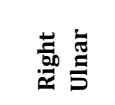 & 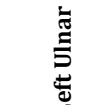 & 㺃离 & 可 & 营施 & 氖 \\
\hline \multirow{3}{*}{ All } & $\mathrm{N}$ & 50 & 50 & 50 & 50 & 50 & 50 & 50 & 50 \\
\hline & Mean & 15.32 & 13.52 & 10.62 & 9.95 & 6.47 & 6.69 & 13.99 & 14.46 \\
\hline & SD & 11.50 & 4.57 & 2.46 & 2.31 & 2.32 & 2.14 & 5.34 & 5.11 \\
\hline \multirow{3}{*}{ Hypothyroid } & $\mathrm{N}$ & 37 & 37 & 37 & 37 & 37 & 37 & 37 & 37 \\
\hline & Mean & 15.58 & 13.90 & 10.66 & 9.81 & 6.71 & 6.93 & 14.30 & 14.36 \\
\hline & SD & 12.94 & 4.58 & 2.37 & 2.30 & 2.44 & 1.98 & 5.62 & 5.25 \\
\hline \multirow{3}{*}{ Hyperthyroid } & $\mathrm{N}$ & 13 & 13 & 13 & 13 & 13 & 13 & 13 & 13 \\
\hline & Mean & 14.58 & 12.43 & 10.52 & 10.36 & 5.78 & 6.01 & 13.10 & 14.74 \\
\hline & SD & 5.08 & 3.52 & 2.42 & 2.08 & 1.42 & 2.12 & 4.12 & 4.48 \\
\hline \multicolumn{2}{|c|}{ P value Unpaired ' $\mathrm{t}$ ' Test } & 0.0140 & 0.0253 & 0.0168 & 0.0411 & 0.0027 & 0.0479 & 0.0493 & 0.0169 \\
\hline \multicolumn{10}{|c|}{ Table 3: Statistics of CMAP of both Upper and Lower Limb Nerves } \\
\hline
\end{tabular}




\begin{tabular}{|c|c|c|c|}
\hline Mean CMAP (Amplitude (mv)) & Hypothyroid & Hyperthyroid & Overall \\
\hline Median & 14.74 & 13.50 & 14.12 \\
\hline Ulnar & 10.44 & 10.23 & 10.34 \\
\hline Peroneal & 6.82 & 5.90 & 6.36 \\
\hline Tibial & 14.33 & 13.92 & 14.13 \\
\hline \multicolumn{2}{r}{} \\
\hline
\end{tabular}

\section{RESULTS}

In patients belonging to hypothyroid Group, the mean CMAPmedian is $14.74 \mathrm{mv}$, CMAP - ulnar is $10.23 \mathrm{mv}$, CMAP peroneal is $6.82 \mathrm{mv}$ and CMAP - tibial is $14.33 \mathrm{mv}$. Similarly, in hyperthyroid patients, CMAP- median is $13.50 \mathrm{mv}$, CMAP ulnar is $10.44 \mathrm{mv}$, CMAP - peroneal is $5.90 \mathrm{mv}$ and CMAP tibial is $13.92 \mathrm{mv}$. The increased mean CMAP measurements overall and in hypothyroid group compared to the hyperthyroid Group is statistically significant, as the $p$ value is $<0.05$ as per unpaired t-test indicating a true difference among study groups.
The mean CMAP measurements were meaningfully more overall and especially in Hypothyroid Group compared to the hyperthyroid Group. This significant difference of increased mean CMAP (Mean difference - Median (1.24 mv, 9\%)), Ulnar (0.21 mv, 2\%), Peroneal (0.92 mv, 16\%) and Tibial (0.41 mv, $3 \%$ ), measurement in Hypothyroid Group compared to the hyperthyroid Group is true and has not occurred by chance.

In this study, we can safely conclude that mean CMAP measurements were significantly and consistently higher overall and especially in Hypothyroid Group compared to the hyperthyroid Group.

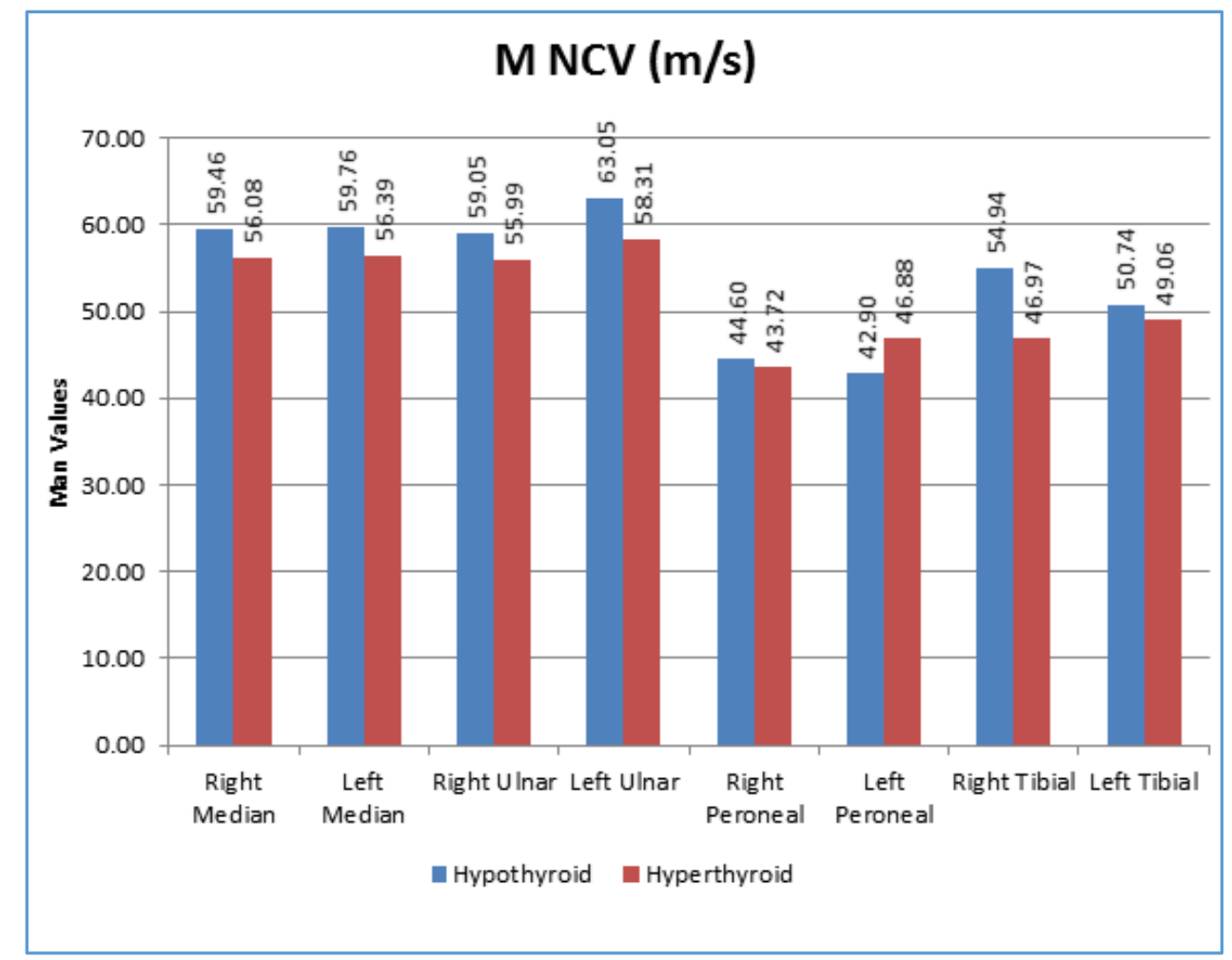

Fig. 5: Motor Nerve Conduction Velocity (MNCV) Bar Diagram Representing Mean MNCV Values of both Upper and Lower Limb Nerves

\begin{tabular}{|c|c|c|c|c|c|c|c|c|c|}
\hline \multicolumn{2}{|c|}{$\operatorname{MNCV}(\mathrm{m} / \mathrm{s})$} & 苞忽 & 屯્ّ & 泀 & 売 & 营苛 & 坖 & 卷 & 带 \\
\hline \multirow{3}{*}{ All } & $\mathrm{N}$ & 50 & 50 & 50 & 50 & 50 & 50 & 50 & 50 \\
\hline & Mean & 58.58 & 58.88 & 58.25 & 61.82 & 44.38 & 43.93 & 52.87 & 50.30 \\
\hline & SD & 5.86 & 6.02 & 5.56 & 7.14 & 4.28 & 6.86 & 23.07 & 10.76 \\
\hline \multirow{3}{*}{ Hypothyroid } & $\mathrm{N}$ & 37 & 37 & 37 & 37 & 37 & 37 & 37 & 37 \\
\hline & Mean & 59.46 & 59.76 & 59.05 & 63.05 & 44.60 & 42.90 & 54.94 & 50.74 \\
\hline & SD & 6.16 & 6.47 & 5.17 & 7.61 & 4.41 & 7.44 & 26.42 & 12.16 \\
\hline \multirow{3}{*}{ Hyperthyroid } & $\mathrm{N}$ & 13 & 13 & 13 & 13 & 13 & 13 & 13 & 13 \\
\hline & Mean & 56.08 & 56.39 & 55.99 & 58.31 & 43.72 & 46.88 & 46.97 & 49.06 \\
\hline & SD & 4.17 & 3.67 & 6.22 & 4.08 & 3.99 & 3.68 & 5.33 & 5.18 \\
\hline \multicolumn{2}{|c|}{$P$ value Unpaired ' $t$ ' Test } & 0.1354 & 0.2277 & 0.1288 & 0.3378 & 0.5122 & 0.4162 & 0.0896 & 0.5002 \\
\hline
\end{tabular}


By conventional criteria, the association between the study groups and MNCV values distribution is considered to be not statistically significant since $\mathrm{p}>0.05$.

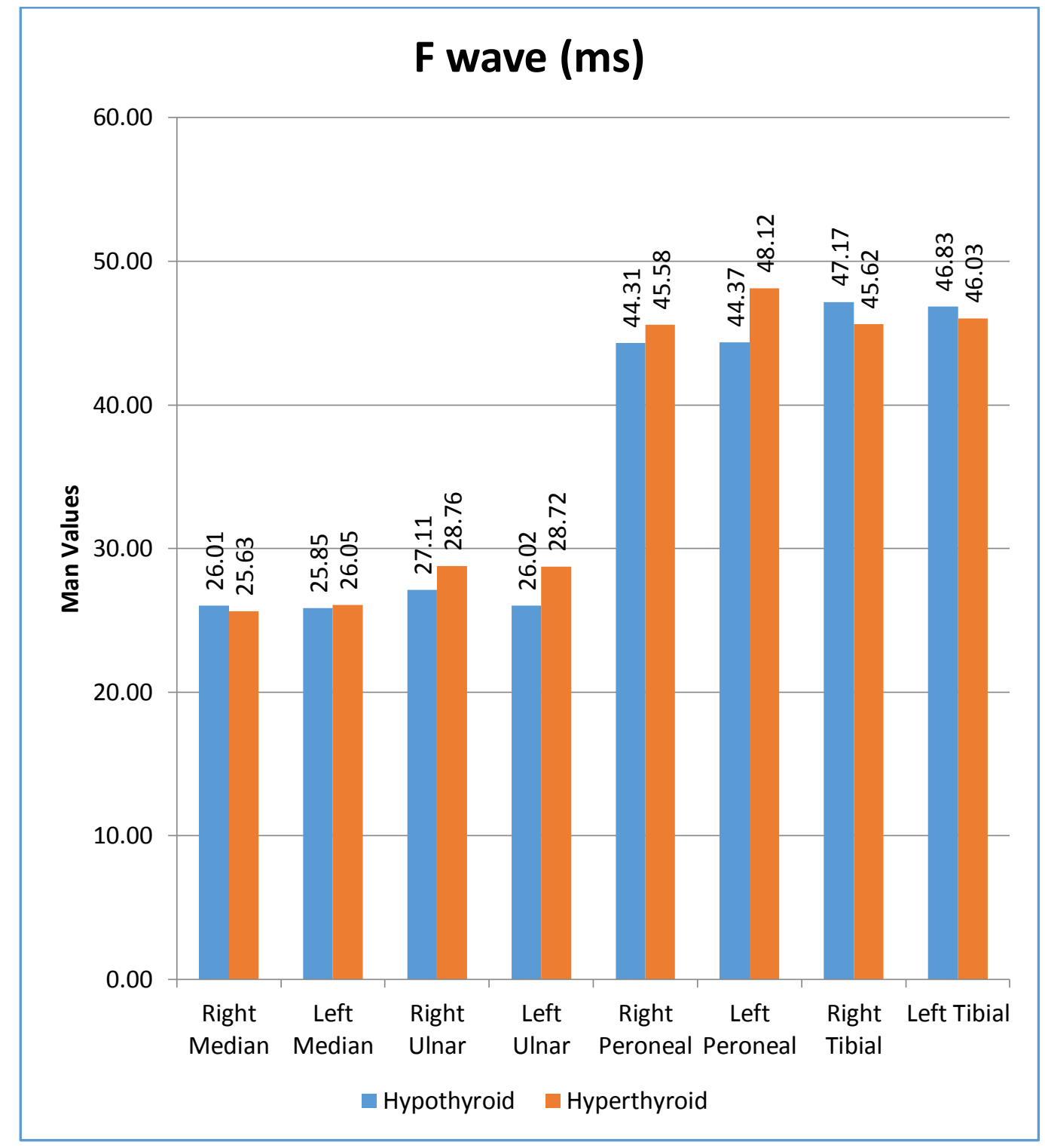

Fig. 6: F Wave (ms) Bar Diagram Representing Mean

F Wave Values of both Upper and Lower Limb Nerves

\begin{tabular}{|c|c|c|c|c|c|c|c|c|c|}
\hline \multicolumn{2}{|c|}{ F Wave (ms) } & 馬 & 屯્屯 & 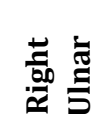 & 茍 壱 & 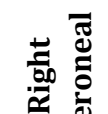 & 密 & 券 & 离 \\
\hline \multirow{3}{*}{ All } & $\mathrm{N}$ & 50 & 50 & 50 & 50 & 50 & 50 & 50 & 50 \\
\hline & Mean & 25.91 & 25.91 & 27.54 & 26.72 & 44.64 & 45.34 & 46.77 & 46.62 \\
\hline & SD & 1.95 & 1.72 & 4.00 & 4.26 & 4.77 & 4.23 & 4.18 & 3.92 \\
\hline \multirow{3}{*}{ Hypothyroid } & $\mathrm{N}$ & 37 & 37 & 37 & 37 & 37 & 37 & 37 & 37 \\
\hline & Mean & 26.01 & 25.85 & 27.11 & 26.02 & 44.31 & 44.37 & 47.17 & 46.83 \\
\hline & SD & 2.01 & 1.83 & 2.25 & 2.22 & 5.11 & 3.82 & 3.08 & 2.67 \\
\hline \multirow{3}{*}{ Hyperthyroid } & $\mathrm{N}$ & 13 & 13 & 13 & 13 & 13 & 13 & 13 & 13 \\
\hline & Mean & 25.63 & 26.05 & 28.76 & 28.72 & 45.58 & 48.12 & 45.62 & 46.03 \\
\hline & SD & 1.81 & 1.38 & 6.94 & 7.31 & 3.63 & 4.24 & 6.39 & 6.40 \\
\hline \multicolumn{2}{|c|}{$P$ value Unpaired ' $t$ ' Test } & 0.5296 & 0.6856 & 0.4155 & 0.2130 & 0.3389 & 0.5110 & 0.4120 & 0.6688 \\
\hline
\end{tabular}

By conventional criteria, the association between the study groups and $\mathrm{F}$ wave values distribution is considered to be not statistically significant since $\mathrm{p}>0.05$. 


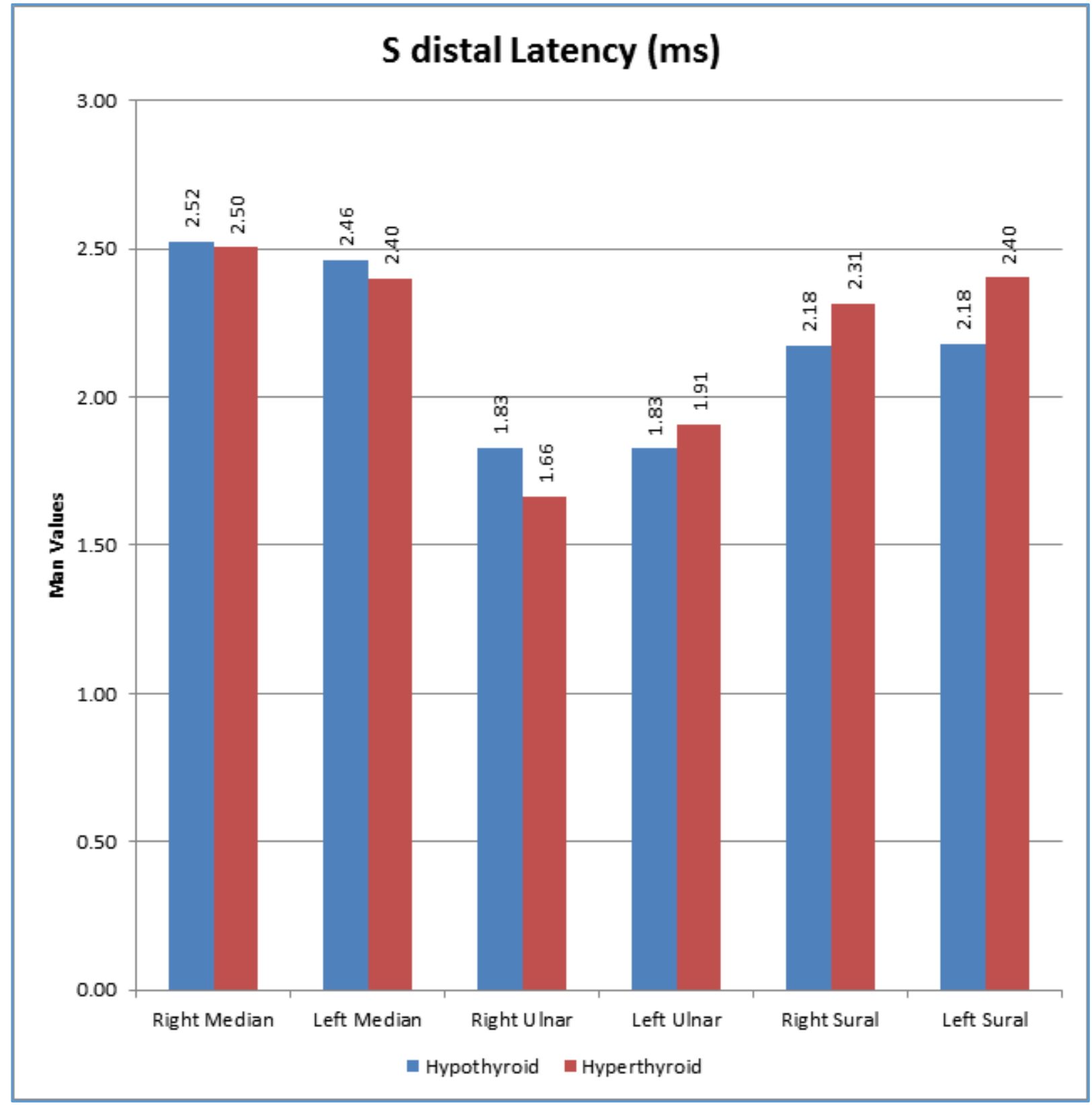

Fig. 7: Sensory Distal Latency (S Distal Latency) Bar Diagram Representing S Distal Latencies of both Upper and Lower Limb Nerves

\begin{tabular}{|c|c|c|c|c|c|c|c|}
\hline \multicolumn{2}{|c|}{ S Distal Latency (ms) } & Right Median & Left Median & Right Ulnar & Left Ulnar & Right Sural & Left Sural \\
\hline \multirow{3}{*}{ All } & $\mathrm{N}$ & 50 & 50 & 50 & 50 & 50 & 50 \\
\hline & Mean & 2.52 & 2.45 & 1.78 & 1.85 & 2.21 & 2.24 \\
\hline & SD & 0.37 & 0.37 & 0.30 & 0.29 & 0.55 & 0.62 \\
\hline \multirow{3}{*}{ Hypothyroid } & $\mathrm{N}$ & 37 & 37 & 37 & 37 & 37 & 37 \\
\hline & Mean & 2.52 & 2.46 & 1.83 & 1.83 & 2.18 & 2.18 \\
\hline & SD & 0.38 & 0.39 & 0.28 & 0.28 & 0.54 & 0.60 \\
\hline \multirow{3}{*}{ Hyperthyroid } & $\mathrm{N}$ & 13 & 13 & 13 & 13 & 13 & 13 \\
\hline & Mean & 2.50 & 2.40 & 1.66 & 1.91 & 2.31 & 2.40 \\
\hline & SD & 0.36 & 0.29 & 0.33 & 0.32 & 0.60 & 0.67 \\
\hline \multicolumn{2}{|c|}{ P value Unpaired ' $\mathrm{t}$ ' Test } & 0.8692 & 0.5633 & 0.1334 & 0.4103 & 0.4718 & 0.3031 \\
\hline & able 7 & f S Distal Lat & cies of both & er and Lou & imb Nerv & & \\
\hline
\end{tabular}

By conventional criteria, the association between the study groups and S distal latency values distribution is considered to be not statistically significant since $\mathrm{p}>0.05$. 


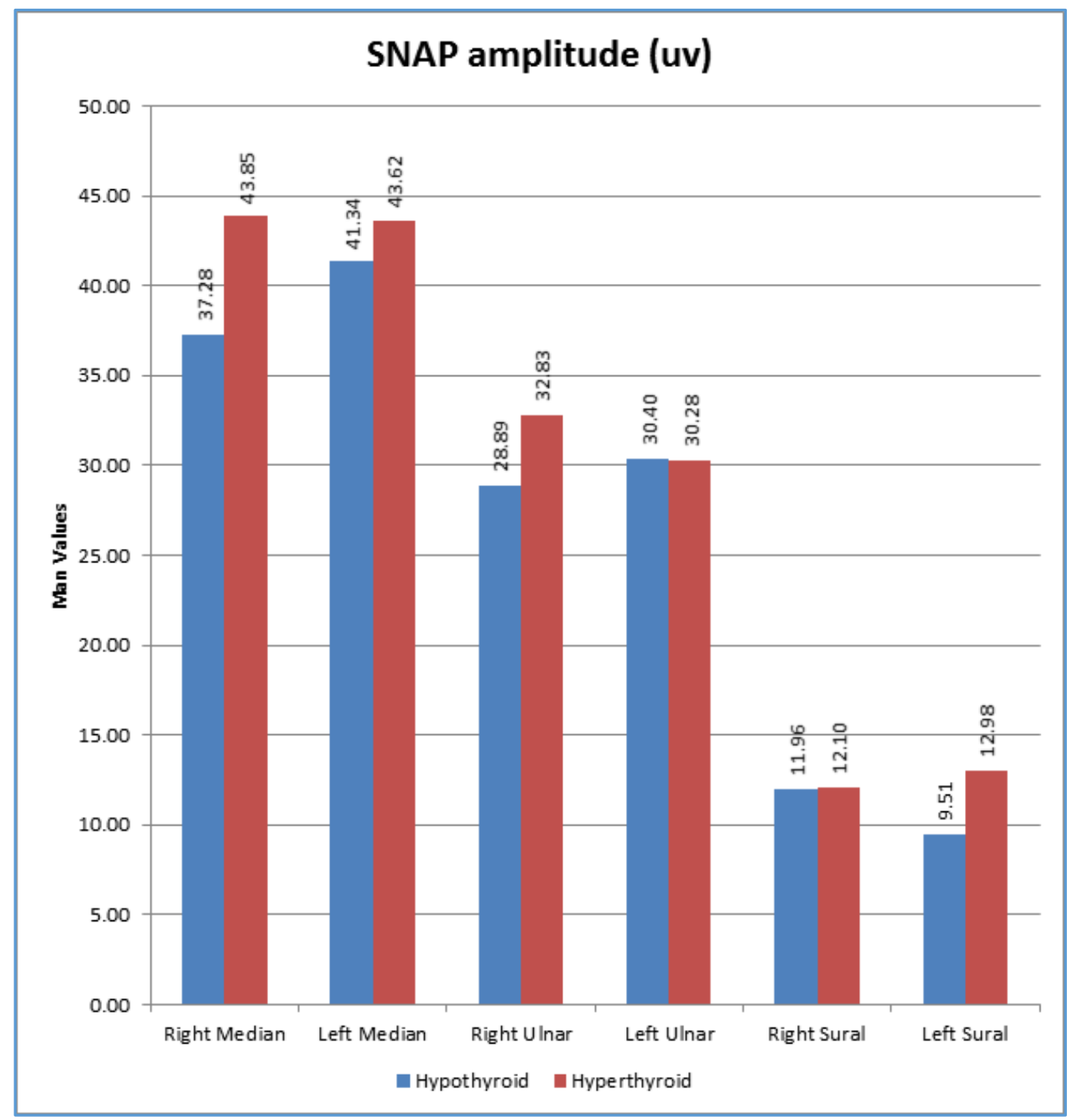

Fig. 8: Sensory Nerve Action Potential (SNAP) Bar Diagram Representing Mean SNAP Amplitude Values of both Upper and Lower Limb Nerves

\begin{tabular}{|c|c|c|c|c|c|c|c|}
\hline \multicolumn{2}{|c|}{ SNAP Amplitude (uv) } & Right Median & Left Median & Right Ulnar & Left Ulnar & Right Sural & Left Sural \\
\hline \multirow{3}{*}{ All } & $\mathrm{N}$ & 50 & 50 & 50 & 50 & 50 & 50 \\
\hline & Mean & 38.99 & 41.93 & 29.92 & 30.37 & 11.99 & 10.41 \\
\hline & SD & 19.89 & 21.25 & 24.86 & 15.98 & 8.81 & 7.59 \\
\hline \multirow{3}{*}{ Hypothyroid } & $\mathrm{N}$ & 37 & 37 & 37 & 37 & 37 & 37 \\
\hline & Mean & 37.28 & 41.34 & 28.89 & 30.40 & 11.96 & 9.51 \\
\hline & SD & 18.41 & 21.55 & 19.32 & 13.73 & 9.15 & 6.56 \\
\hline \multirow{3}{*}{ Hyperthyroid } & $\mathrm{N}$ & 13 & 13 & 13 & 13 & 13 & 13 \\
\hline & Mean & 43.85 & 43.62 & 32.83 & 30.28 & 12.10 & 12.98 \\
\hline & SD & 22.75 & 20.14 & 36.29 & 20.84 & 7.04 & 9.43 \\
\hline \multicolumn{2}{|c|}{ P value Unpaired ' $\mathrm{t}$ ' Test } & 0.0370 & 0.0420 & 0.0213 & 0.0147 & 0.0046 & 0.0226 \\
\hline
\end{tabular}

\begin{tabular}{|c|c|c|c|}
\hline SNAP Amplitude & Hypothyroid & Hyperthyroid & Overall \\
\hline Median & 39.31 & 43.74 & 41.52 \\
\hline Ulnar & 29.65 & 31.55 & 30.60 \\
\hline Sural & 10.73 & 12.54 & 11.64 \\
\hline \multicolumn{2}{|c|}{ Table 9: Overall Mean SNAP Values in Thyroid Dysfunction } \\
\hline
\end{tabular}

\section{RESULTS}

In patients belonging to hypothyroid Group, the mean SNAPmedian is 39.31 uv, SNAP- ulnar is 29.65 uv and SNAPsuralis 10.73 uv. Similarly, in hyperthyroid patients SNAPmedian is 43.74 uv. SNAP - ulnar is 31.55 uv and SNAP -sural is $12.54 \mathrm{uv}$. The increased mean SNAP measurements overall and decreased in hypothyroid group compared to the hyperthyroid Group is statistically significant, as the $\mathrm{p}$ value is $<0.05$ as per unpaired t-test indicating a true difference among study groups. 
The mean SNAP measurements were meaningfully more overall and less in Hypothyroid Group compared to the hyperthyroid Group. This significant difference of decreased mean.

SNAP (Mean difference-Median (4.43 nv, 10\%), Ulnar (1.91 mv, 6\%) and Sural (1.81 mv, 14\%), measurement in
Hypothyroid Group compared to the hyperthyroid Group is true and has not occurred by chance.

In this study, we can safely conclude that mean SNAP measurements were significantly and consistently higher overall and especially lowered in Hypothyroid Group compared to the hyperthyroid Group.

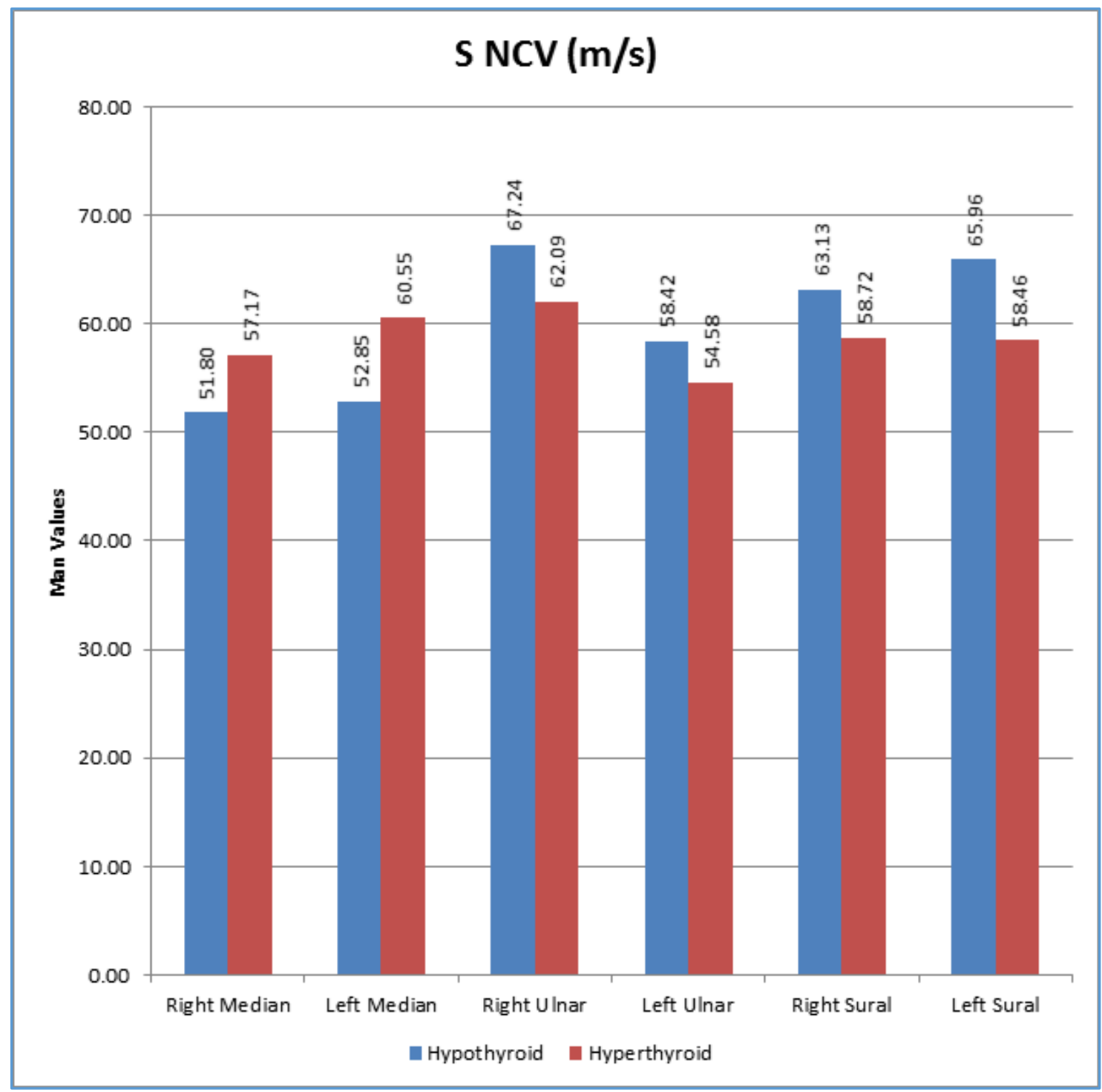

Fig. 9: Sensory Nerve Conduction Velocity (SNCV) Bar Diagram Representing the Mean SNCV Values of both Upper and Lower Limb Nerves

\begin{tabular}{|c|c|c|c|c|c|c|c|}
\hline \multicolumn{2}{|c|}{ SNCV $(\mathrm{m} / \mathrm{s})$} & Right Median & Left Median & Right Ulnar & Left Ulnar & Right Sural & Left Sural \\
\hline \multirow{3}{*}{ All } & $\mathrm{N}$ & 50 & 50 & 50 & 50 & 50 & 50 \\
\hline & Mean & 53.20 & 54.85 & 65.90 & 57.42 & 61.99 & 64.01 \\
\hline & SD & 8.91 & 8.56 & 56.80 & 7.39 & 12.81 & 13.77 \\
\hline \multirow{3}{*}{ Hypothyroid } & $\mathrm{N}$ & 37 & 37 & 37 & 37 & 37 & 37 \\
\hline & Mean & 51.80 & 52.85 & 67.24 & 58.42 & 63.13 & 65.96 \\
\hline & SD & 8.99 & 7.97 & 65.75 & 8.04 & 13.66 & 12.97 \\
\hline \multirow{3}{*}{ Hyperthyroid } & $\mathrm{N}$ & 13 & 13 & 13 & 13 & 13 & 13 \\
\hline & Mean & 57.17 & 60.55 & 62.09 & 54.58 & 58.72 & 58.46 \\
\hline & SD & 7.65 & 7.81 & 13.48 & 4.11 & 9.70 & 15.01 \\
\hline \multicolumn{2}{|c|}{ P value Unpaired ' $t$ ' Test } & 0.1482 & 0.2262 & 0.6547 & 0.3334 & 0.2172 & 0.1256 \\
\hline
\end{tabular}

By conventional criteria, the association between the study groups and SNCV distribution is considered to be not statistically significant since $\mathrm{p}>0.05$. 


\section{Type of Neuropathy}

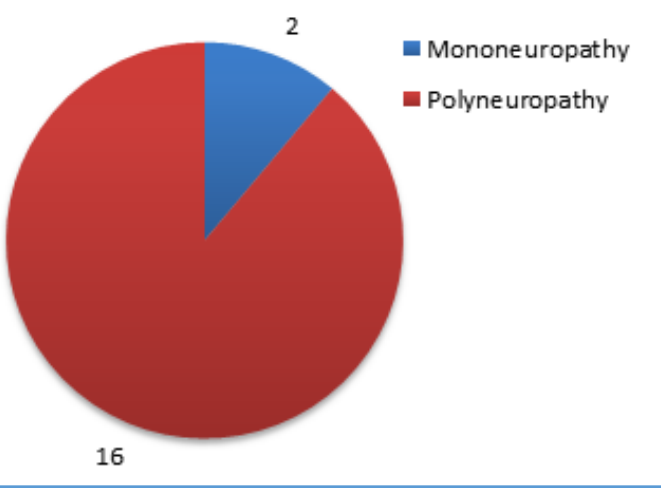

Fig. 10: Pie Chart Representing the Type of Neuropathy among the Study Population

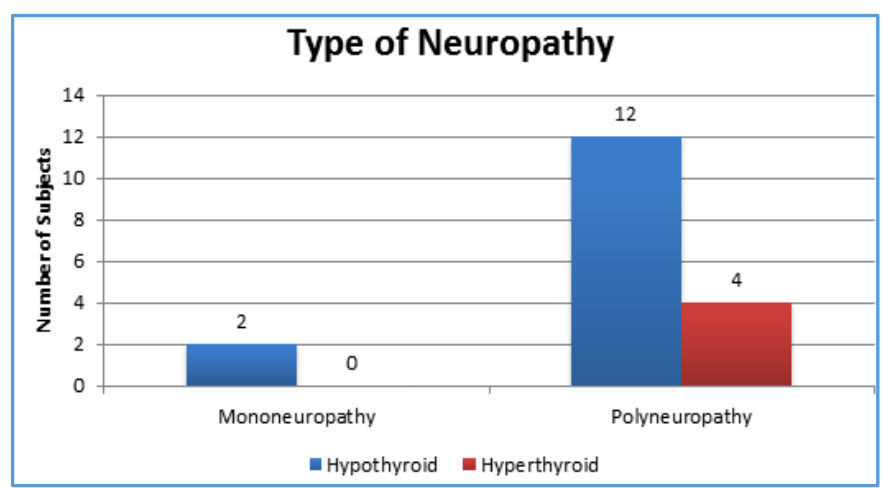

Fig. 11: Bar Diagram Representing the Frequency Distribution of Type of Neuropathy in both Hyper and Hypothyroid Group

\begin{tabular}{|c|c|c|c|c|c|c|}
\hline Type of Neuropathy & All & $\%$ & Hypothyroid & $\%$ & Hyperthyroid & $\%$ \\
\hline Mononeuropathy & 2 & 4.00 & 2 & 5.41 & 0 & 0.00 \\
\hline Polyneuropathy & 16 & 32.00 & 12 & 32.43 & 4 & 30.77 \\
\hline Total & 18 & 36 & 14 & 38 & 4 & 31 \\
\hline \multicolumn{3}{|c|}{ P value Fisher's Exact Test } & \multicolumn{4}{|c|}{0.9999} \\
\hline
\end{tabular}

By conventional criteria, the association between the study groups and type of neuropathy is considered to be not statistically significant since $\mathrm{p}>0.05$.

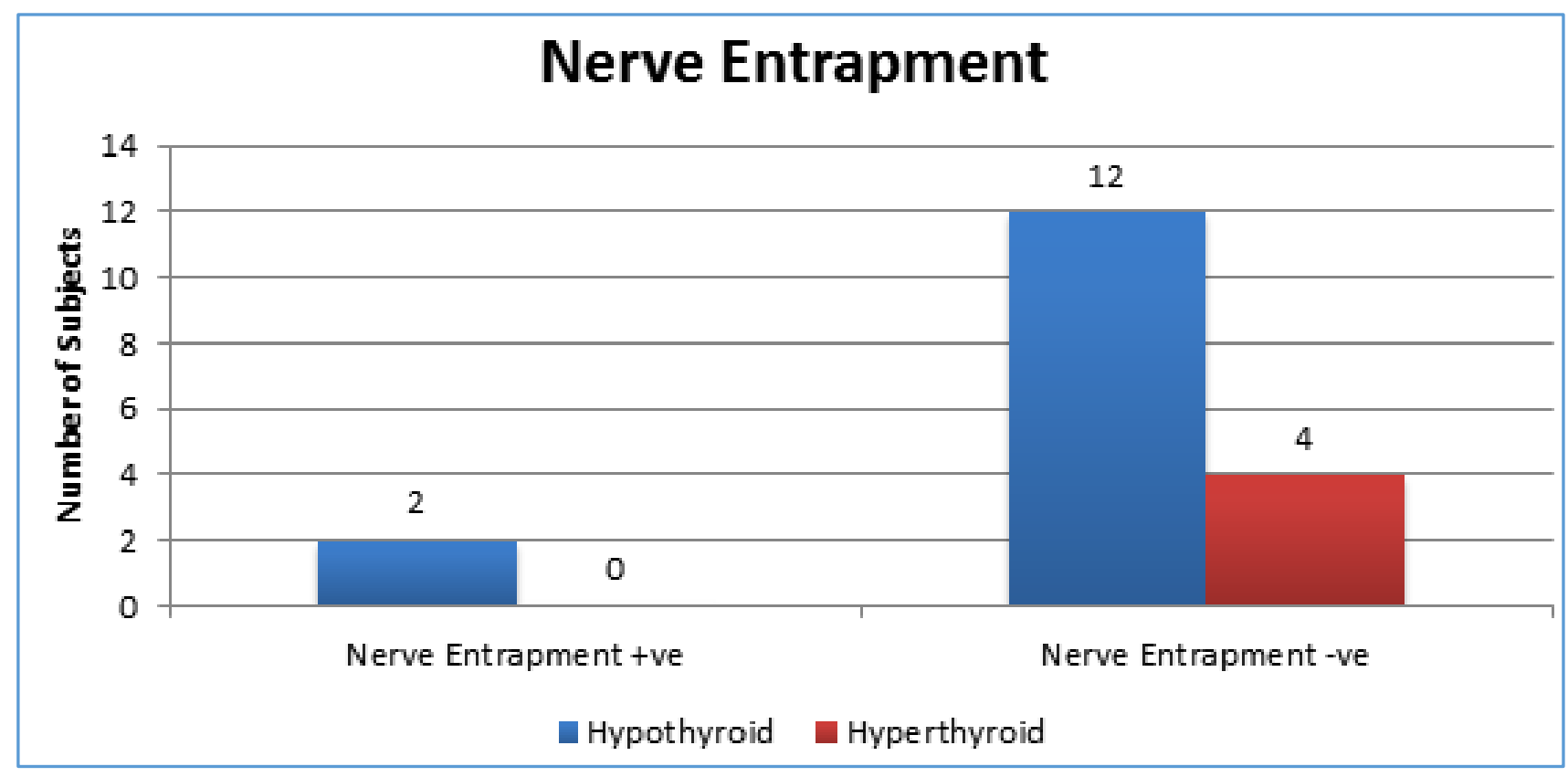

Fig. 12: Bar Diagram Depicting Number of Patients Found to have Nerve Entrapment among both Hyper and Hypothyroid Group

\begin{tabular}{|c|c|c|c|c|c|c|}
\hline Nerve Entrapment & All & \% & Hypothyroid & \% & Hyperthyroid & \% \\
\hline Nerve Entrapment +ve & 2 & 11.11 & 2 & 14.29 & 0 & 0.00 \\
\hline Nerve Entrapment -ve & 16 & 88.89 & 12 & 85.71 & 4 & 100.00 \\
\hline Total & 18 & 100 & 14 & 100 & 4 & 100 \\
\hline P value Fisher's Exact Test & & \multicolumn{2}{|c|}{0.9999} \\
\hline
\end{tabular}

By conventional criteria the association between the study groups and nerve entrapment is considered to be not statistically significant since $\mathrm{p}>0.05$. 


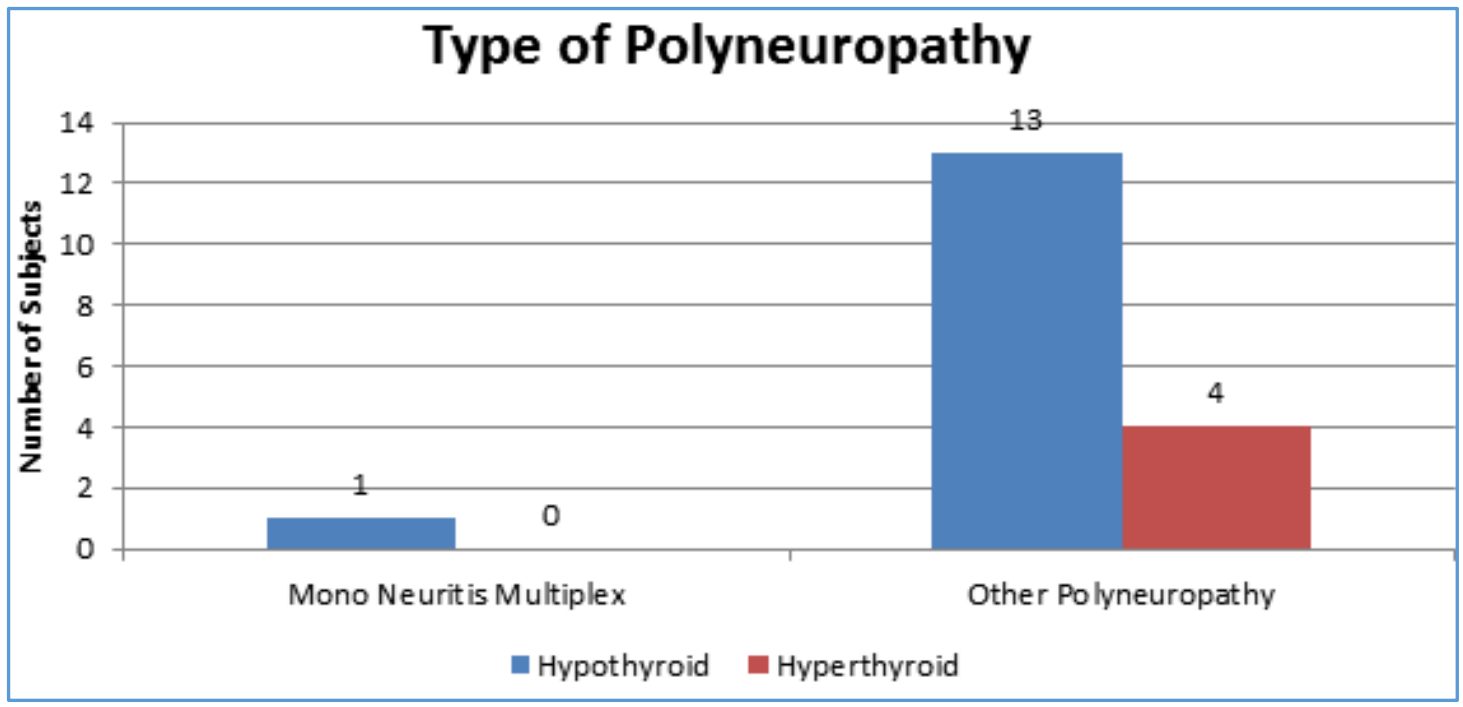

Fig. 13: Bar Diagram Explaining the Types of Polyneuropathy among Study Population

\begin{tabular}{|c|c|c|c|c|c|c|}
\hline Type of Polyneuropathy & All & $\mathbf{\%}$ & Hypothyroid & $\mathbf{\%}$ & Hyperthyroid & \% \\
\hline Mononeuritis Multiplex & 1 & 11.11 & 1 & 100.00 & 0 & 76.47 \\
\hline Other Polyneuropathy & 17 & 88.89 & 13 & 0.00 & 23.53 \\
\hline Total & 18 & 100 & 1 & 100 & 17 & 100 \\
\hline P value Fisher's Exact Test & \multicolumn{4}{|c|}{0.9999} \\
\hline
\end{tabular}

By conventional criteria, the association between the study groups and type of polyneuropathy is considered to be not statistically significant since $\mathrm{p}>0.05$.

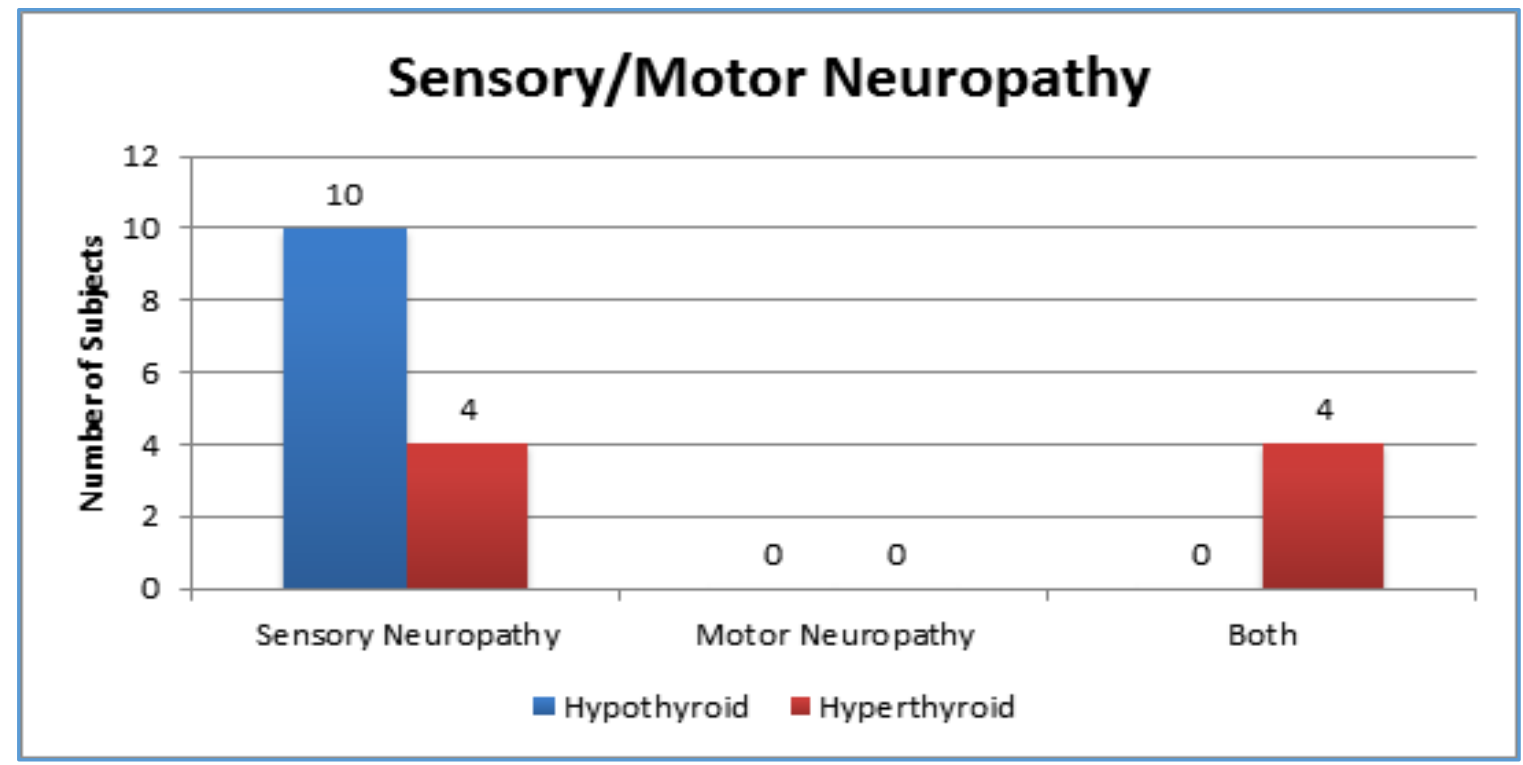

Fig. 14: Bar Diagram Depicting the Presence of either Sensory, Motor Neuropathy or Both among Study Population

\begin{tabular}{|c|c|c|c|c|c|c|}
\hline Sensory/Motor Neuropathy & All & $\%$ & Hypothyroid & $\%$ & Hyperthyroid & $\%$ \\
\hline Sensory Neuropathy & 14 & 77.78 & 10 & 100.00 & 4 & 50.00 \\
\hline Motor Neuropathy & 0 & 0.00 & 0 & 0.00 & 0 & 0.00 \\
\hline Both & 4 & 22.22 & 0 & 0.00 & 4 & 50.00 \\
\hline Total & 18 & 100 & 10 & 100 & 8 & 100 \\
\hline \multicolumn{3}{|c|}{ P value Fisher's Exact Test } & \multicolumn{4}{|c|}{0.0229} \\
\hline
\end{tabular}




\section{RESULTS}

In patients belonging to hypothyroid group, the majority had sensory neuropathy $(\mathrm{n}=10,100 \%)$. In hyperthyroid group, the majority had sensory neuropathy $(n=4,50 \%)$. The increased incidence of sensory neuropathy in hypothyroid group compared to hyperthyroid group is statistically significant, as the p value is 0.0229 as per Fisher's exact test indicating a true difference among study groups.

The incidence of sensory neuropathy was meaningfully more in hypothyroid group compared to hyperthyroid group by 50.00 percentage points. This significant difference of 2 times increase in incidence of sensory neuropathy in hypothyroid group compared to hyperthyroid group is true and has not occurred by chance.

In this study, we can safely conclude that incidence of sensory neuropathy was significantly and consistently higher in hypothyroid group compared to hyperthyroid group.

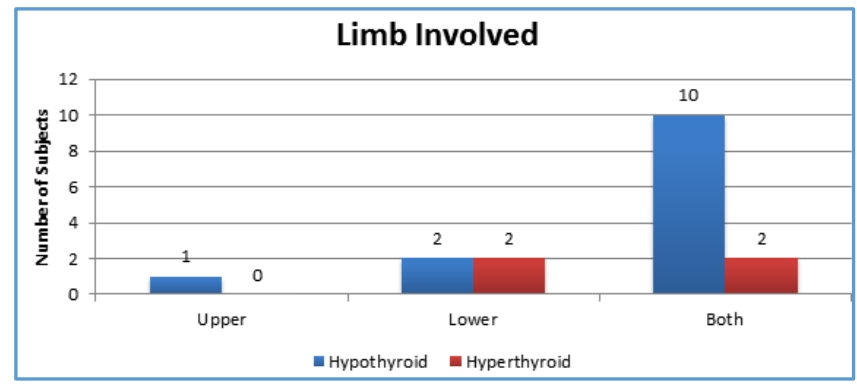

Fig. 15: Bar Diagram Representing the Limbs Involved among the Study Population

\begin{tabular}{|c|c|c|c|c|c|c|}
\hline 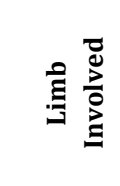 & $\bar{z}$ & $0^{\circ}$ & 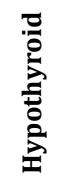 & $0^{\circ}$ & 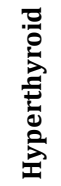 & $0^{\circ}$ \\
\hline Upper & 1 & 5.88 & 1 & 7.69 & 0 & 0.00 \\
\hline Lower & 4 & 23.53 & 2 & 15.38 & 2 & 50.00 \\
\hline Both & 13 & 70.59 & 11 & 76.92 & 2 & 50.00 \\
\hline Total & 18 & 100 & 13 & 100 & 4 & 100 \\
\hline \multicolumn{3}{|c|}{ P value Fisher's Exact Test } & \multicolumn{4}{|c|}{0.3295} \\
\hline
\end{tabular}

By conventional criteria, the association between the study groups and limb involvement is considered to be not statistically significant since $\mathrm{p}>0.05$.

\section{RESULTS}

\section{Following are the Results from our Study}

1. Majority of the hypothyroid Group patients belonged to the 31-40 years age class interval $(n=13,35.14 \%)$ with a mean age of 35.37 years. In the hyperthyroid group patients, majority belonged to the $\leq 30$ years age class interval $(n=6,46.15 \%)$ with a mean age of 34.77 years.

2. Majority of the hypothyroid Group patients belonged to female gender, class interval $(n=28,75.68)$. In the hyperthyroid group patients, majority belonged to the female gender class interval $(n=7,53.85 \%)$.

3. Majority of the hypothyroid Group patients belonged to the 7-12 months' duration of symptoms class interval $(\mathrm{n}=14,37.84)$. In the hyperthyroid group patients, majority belonged to the 4-6 months' duration of symptoms class interval $(n=7,53.85 \%)$.

4. In patients belonging to hypothyroid Group, the mean CMAP - median is $14.74 \mathrm{mv}$, CMAP - ulnar is $10.23 \mathrm{mv}$, CMAP - peroneal is $6.82 \mathrm{mv}$ and CMAP - tibial is 14.33 mv. Similarly in hyperthyroid patients, CMAP- median is $13.50 \mathrm{mv}$, CMAP - ulnar is $10.44 \mathrm{mv}$, CMAP - peroneal is $5.90 \mathrm{mv}$ and CMAP-tibial is $13.92 \mathrm{mv}$. The increased mean CMAP measurements overall and in hypothyroid group compared to the hyperthyroid Group is statistically significant, as the $p$ value is $<0.05$ as per unpaired t-test indicating a true difference among study groups.

5. In patients belonging to hypothyroid Group, the mean SNAP- median is $39.31 \mathrm{uv}$, SNAP - ulnar is $29.65 \mathrm{uv}$ and SNAP- suralis 10.73 uv. Similarly, in hyperthyroid patients SNAP- median is $43.74 \mathrm{uv}$, SNAP- ulnar is 31.55 uv and SNAP- sural is $12.54 \mathrm{uv}$. The increased mean SNAP measurements overall and decreased in hypothyroid group compared to the hyperthyroid group is statistically significant as the $p$ value is $<0.05$ as per unpaired t-test indicating a true difference among study groups.

6. By conventional criteria, the association between the study groups and various parameters like $\mathrm{M}$ distal latency, M NCV, F wave, S distal latency, S NCV are considered to be not statistically significant since $p$ $>0.05$.

- $\quad$ Out of the 50 patients studied, 18 patients had neuropathy. Of which 14 of them were found to be hypothyroid and 4 of them had hyperthyroidism; 2 patients had mononeuropathy and 16 of them had polyneuropathy.

- Entrapment features like carpal tunnel syndrome was present in 2 hypothyroid individuals and 1 patient had features of mononeuritis multiplex.

- 14 patients had predominantly sensory neuropathy and 4 individuals had both sensory and motor polyneuropathy.

- 13 patients had both upper limb and lower limb involvement.

- 4 patients had predominantly lower limb involvement and 1 patient had predominantly upper limb involvement.

- $\quad$ The amplitude of CMAP and SNAP were particularly altered in both groups and it was also statistically significant. Thereby, it reflects the axonal pattern of sensory loss, which is expected in thyroid illness.

\section{DISCUSSION}

Hypothyroidism Patients can develop 2 types of Peripheral Neuropathy. They are:

1. Sensorimotor polyneuropathy due to axonal and myelin changes. It has distoproximal progression affecting lower limbs first followed by upper limbs. The severity of this neuropathy is related to the duration of the disease than the deficiency of hormone levels.

2. Mononeuropathy-entrapment syndromes due to mucinous deposits that compress the nerves.

In hyperthyroidism, neuropathy is usually an uncommon manifestation. Acute neuropathy associated with paraplegia 
has been reported as Basedow's paraplegia. The possible mechanisms quoted are direct effect of thyroid hormone, immune mediated and hypermetabolic state depleting the nerves of essential substances. Motor neuron stoma is the site of primary lesion. Carpal Tunnel Syndrome (CTS) can be a manifestation of hyperthyroidism. Our study concludes that hypothyroid individuals are more predisposed to develop sensory motor axonal polyneuropathy. The same was quoted by many authors as follows:

1. Duffy et al quotes.(1) $19 \%$ of hyperthyroid individuals had sensory axonal neuropathy, which matches our study that $30.7 \%$ of hyperthyroid individuals had it.

2. Our study did not show any F wave changes in median and peroneal nerves; this is in contrary to Udhaya Kumar et al.

3. O'Malley et al(2) and our study is similar in showing sensory threshold abnormalities in multiple nerves.

4. Schutt et al(3) clearly demonstrated motor conduction abnormalities, whereas our study contradicts it by having predominant sensory conduction abnormalities.

\section{CONCLUSION}

The most common neurological abnormalities encountered among the study population were sensory axonal polyneuropathy.

- Mononeuropathy involving the sural nerve.

- Mononeuritis multiplex pattern.

- Carpal tunnel syndrome.
To conclude hypothyroid patients are more prone to develop neuropathy, predominantly involving the sensory nerves in lower limbs. Electrophysiological studies can be useful in the diagnosis of subclinical polyneuropathy that is more prevalent in newly detected thyroid dysfunction.

Henceforth, nerve conduction studies (Electrophysiological study) can be included in the early part of diagnostic workup panel in newly detected thyroid illness. Since this neuropathy at early stage is reversible, it can also be used to test the prognosis of hypothyroidism and hyperthyroidism on Standard treatment.

\section{REFERENCES}

1. Duyff RD, Bosh JV, Laman DM, et al. Neuromuscular findings in thyroid dysfunctions: a prospective clinical and electrodiagnostic study. J Neurol Neurosurg Psychiatry 2000;68(6):750-5.

2. O'Malley BP, Abbott RJ, Timson L, et al. Sensory thresholds and motor responsiveness in thyroid disease: their responses to treatment and warming. Clin Endocrinol (Oxf) 1985;23(1):17-23.

3. Schutt P, Muche H, Gallenkamp U, et al. Reversible alterations of peripheral nerve function in thyroid dysfunction. EEG EMG Z Elektroenzephalogr Elektromyogr Verwandte Geb 1979;10(2):101-5. 